\title{
Evaluation of Changes in Bone Density and Biochemical Parameters after Parathyroidectomy in Primary Hyperparathyroidism
}

\author{
DAIKI NAKAOKA, Toshitsugu SUGIMOTO, TATSUYA KOBAYASHI, TORU YAMAGUCHI, \\ AKIRA KOBAYASHI* AND KaZUO CHIHARA
}

Third Division, Department of Medicine, Kobe University School of Medicine, Kobe 650-0017, Japan

* Kuma Hospital, Kobe 650-0011, Japan

\begin{abstract}
We examined the relationships between serum levels of intact parathyroid hormone (PTH) and alkaline phosphatase (ALP) versus bone mineral density (BMD) at the lumbar spine and radius in terms of their preoperative values and of their annual percentage and net changes after parathyroidectomy (PTX) in 44 Japanese patients (14 men and 30 women) with primary hyperparathyroidism (pHPT). Lumbar and radial BMD values were measured by dual energy X-ray absorptiometry and single photon absorptiometry and were used for evaluating the cancellous and cortical bone mass, respectively. Age- and sex-adjusted value (Z-score) of the radial BMD was significantly lower than that of the lumbar BMD before and after PTX $(P<0.05)$. In preoperative patients, serum levels of both intact PTH and ALP were significantly and negatively correlated with $\mathrm{Z}$-score of the radial $\mathrm{BMD}(\mathrm{P}<0.05$ and $\mathrm{P}<0.001$, respectively), but not with that of the lumbar BMD. After PTX, serum levels of calcium, phosphorus, ALP, and PTH became normal, and both lumbar and radial BMD values markedly increased over 1 year, with percentage changes of $12.2 \pm 1.4 \%$ and $11.6 \pm 1.6 \%$, respectively, which were larger than those in any other Caucasian study previously documented. Even in patients without osteopenia (Z-score of BMD $\geq 0$ ), lumbar and radial BMD values increased considerably after the operation $(9.6 \pm 1.9 \%$ and $6.7 \pm 1.4 \%$, respectively). Annual percentage and net changes in lumbar BMD were significantly and negatively correlated with those in ALP with high correlation coefficients, but those in radial BMD were correlated only with the annual net change in ALP but not with the percentage change. No significant correlations were observed between annual changes in either lumbar or radial BMD and those in intact PTH. Taken together, this study shows that PTX causes dramatic improvements in both the cancellous and cortical bone mass in Japanese pHPT patients regardless of the severity of their osteopenia, and suggests that the cancellous and cortical bones react differently to a preoperative endogenous PTH excess and a high bone turnover rate as well as to the postoperative normalization of a bone turnover rate in the patients.
\end{abstract}

Key words: Primary hyperparathyroidism, Parathyroid hormone, Bone mineral density, Parathyroidectomy

(Endocrine Journal 47: 231-237, 2000)

THE increasing availability of inexpensive and accurate measurements of serum calcium has raised awareness of primary hyperparathyroidism (pHPT) without overt symptoms in physicians [1]. pHPT is

Received: June 10, 1999

Accepted: March 6, 2000

Correspondence to: Dr. Toshitsugu SUGIMOTO, Third Division, Department of Medicine, Kobe University School of Medicine, 7-5-1 Kusunoki-cho, Chuo-ku, Kobe 650-0017, Japan often cited as one of the diseases that cause secondary osteoporosis especially in the cortical bone due to high sustained circulating levels of parathyroid hormone (PTH), which is known to have a catabolic action and to stimulate osteoclastic bone resorption and bone turnover $[2,3]$. On the other hand, several lines of evidence show that PTH also has an anabolic action on bone in certain circumstances $[4,5]$. Clinical trials in patients with osteoporosis and experiments with osteopenic animals have shown that intermittent administration of PTH causes an in- 
crease in the cancellous bone mass [6-8], and in patients with mild pHPT, several studies have revealed that the cancellous bone mass is rather preserved $[3,9,10]$, suggesting that a mildly elevated PTH level may have a protective effect on the cancellous bone.

The cortical and cancellous bones also seem to react differently to the normalization of a high circulating level of PTH and of a high bone turnover rate which occurred after parathyroidectomy (PTX) in pHPT patients. Bone mineral density (BMD) has been reported to increase after PTX in Caucasian pHPT patients [11-16], and two studies have shown that the percentage increase in BMD of the lumbar spine after PTX was greater than that in BMD of the radius $[16,17]$. However, such clinical studies have yet to be performed in Japanese pHPT patients, and thus it remains unclear whether or not PTX has a similar beneficial effect on bone mass, or whether or not the recovery of bone loss after PTX is different between the cortical and cancellous bones in Japanese patients. In the present study, we assessed the cortical and cancellous bone mass in Japanese pHPT patients by radial and lumbar BMD values, respectively, and followed their changes over 1 year after PTX. We also analyzed the relationships between these BMD values versus serum levels of intact PTH and ALP in terms of their preoperative values and their annual percentage and net changes after PTX.

\section{Subjects and Methods}

\section{Patients}

Background data on $103 \mathrm{pHPT}$ patients in our clinic have been reported previously [18]. Among them, we analyzed 44 patients (14 men [mean age 54] and 30 women [mean age 58]) who were admitted to Kuma Hospital or Kobe University Hospital for PTX and were followed by BMD measurements before and 1 year after the operation during 1989 to 1997. Sixteen patients had a history of nephrolithiasis and 4 patients had vertebral fractures by lateral thoracic and lumbar spine radiographs. None of the patients had hepatic or renal dysfunction or other metabolic diseases that might cause secondary changes in bone metabolism. The patients had not taken any drugs known to influence bone mineral metabolism. The clinical and biochemical data were obtained by reviewing the chart records at admission and 1 year after PTX. This study was approved by the Ethical Review Board of Kobe University Hospital and was performed under the informed consent of patients.

\section{Biochemical measurements}

Serum concentrations of calcium, phosphorus, and alkaline phosphatase (ALP) were measured by automated techniques (normal range: calcium 8.4$9.9 \mathrm{mg} / \mathrm{dl}$, phosphorus $2.4-4.5 \mathrm{mg} / \mathrm{dl}$, ALP 100 $303 \mathrm{IU} / \mathrm{l})$. Intact PTH was measured by an immunoradiometric assay (Allegro Intact PTH-RIA kit, Nichols Institute Diagnostics, San Juan Capistrano, CA; normal range: $10-65 \mathrm{pg} / \mathrm{ml}$ ) [19].

\section{BMD measurements}

BMD of the lumbar spine at L2-L4 in the anteroposterior view (lumbar BMD) was measured at admission and one year after PTX by dual energy X-ray absorptometry (DXA) (Hologic QDR 1000, Waltham, MA). The patients who had vertebral fractures at the lumbar spine (L2-L4) by radiographs were excluded from the study. Bone mineral content (BMC), bone width (BW), and BMD (defined as $\mathrm{BMC} / \mathrm{BW}$ ) were measured at the distal one-third of the radius (radial BMD) using single photon absorptiometry (SPA) (Bone Mineral Analyzer Type 278 O, Norland Corp., Fort Atkinson, WI). Values were also expressed relatively as standard deviation (SD) of a sex-, age-, and weight-adjusted normal Japanese mean value (Z-score) and as SD of a mean value of peak bone mass in the normal young adult Japanese population (T-score) in each sex. The coefficients of vatiation (precision) of measurements of the lumbar BMD and the radial BMD by our methods were $0.9 \%$ and $1.9 \%$, respectively.

\section{Statistical analysis}

All data were expressed as the mean \pm SEM. Statistical significance was determined using Student's $t$-test. The regression analysis was performed using the statistical computer program StatView (Abacus Concepts, Inc., Berkeley, CA). Simple regression analysis was used to assess the linear 
relationship between study parameters, and then Pearson's correlation coefficients were calculated. $\mathrm{P}$-values less than 0.05 were considered significant.

\section{Results}

Table 1 compares patient states at admission and 1 year after PTX with respect to age, biochemical and BMD parameters. All patients had elevated serum levels of calcium, ALP, and intact PTH at admission. These values became normal after PTX, indicating that the operations were performed successfully. Both $\mathrm{Z}$ and $\mathrm{T}$ scores of the radial BMD were significantly lower than those of the lumbar BMD at admission $(\mathrm{P}<0.05$ and $\mathrm{P}<0.01$, respectively). There was no difference in $\mathrm{Z}$ scores of radial and lumbar BMD values between men and women (data not shown). Preoperative serum levels of both intact PTH and ALP were significantly and negatively correlated with preoperative $\mathrm{Z}$ score of the radial BMD $(\mathrm{r}=-0.295, \mathrm{P}<0.05$ and $\mathrm{r}=-0.388, \mathrm{P}<0.001$, respectively), but not with that of the lumbar BMD. Both radial and lumbar BMD values increased considerably after PTX, although they were still lower than normal, judging from their below-zero $\mathrm{Z}$ scores after the operation.
Fig. 1 shows changes in radial and lumbar BMD values over the 1-year measurement period in the patients. All subjects showed improvements in BMD values at both sites after PTX. The annual percentage and net changes in radial BMD values were $11.6 \pm 1.6 \%$ (mean $\pm \mathrm{SE}$, range 0.3-46.9) and $0.048 \pm 0.006 \mathrm{~g} / \mathrm{cm}^{2}(0.002-0.143)$, respectively, and those in lumbar BMD values were $12.2 \pm 1.4 \%(0.5-$ $40.8)$ and $0.077 \pm 0.007 \mathrm{~g} / \mathrm{cm}^{2}(0.005-0.202)$, respectively. The percentage changes in radial and lumbar BMD were positively correlated with each other $(\mathrm{r}=0.51, \mathrm{P}<0.01)$, and there was no significant difference between these two sites in the percentage change in BMD after PTX. There were no sexdifferences in the annual percent or net changes in radial or lumbar BMD values (data not shown). The improvements in radial and lumbar BMD values after PTX were found even in a subset of patients without osteopenia (preoperative $\mathrm{Z}$ score of $\mathrm{BMD} \geq 0$ ) $(6.7 \pm 1.4 \%$ and $9.6 \pm 1.9 \% ; n=9$ and $n=4$, respectively).

We analyzed the correlations between the percentage and net changes in BMD values versus those in intact PTH and ALP after PTX (Table 2). The percentage and net changes in lumbar BMD were significantly and negatively correlated with those in ALP with high correlation coefficients, but those in

Table 1. Comparison of preoperative and one year-postoperative states in patients with primary hyperparathyroidism receiving parathyroidectomy

\begin{tabular}{lcc}
\hline & Preoperative & Postoperative \\
\hline Age (years) & $54.2 \pm 1.5$ & $55.2 \pm 1.5$ \\
Calcium (mg/dl) & $11.4 \pm 0.15$ & $9.0 \pm 0.05^{\mathrm{b}}$ \\
Phosphorus (mg/dl) & $2.1 \pm 0.1$ & $3.4 \pm 0.1^{\mathrm{b}}$ \\
Alkaline phosphatase (IU/l) & $559 \pm 95$ & $238 \pm 9^{\mathrm{b}}$ \\
Intact PTH (pg/ml) & $213 \pm 30$ & $41 \pm 5^{\mathrm{b}}$ \\
Radius BMD (g/cm $\left.{ }^{2}\right)$ & $0.462 \pm 0.020$ & $0.511 \pm 0.020^{\mathrm{b}}$ \\
Lumbar spine BMD (g/cm $\left.{ }^{2}\right)$ & $0.705 \pm 0.026$ & $0.787 \pm 0.029^{\mathrm{b}}$ \\
Z score of radius BMD & $-1.92 \pm 0.36$ & $-1.12 \pm 0.34$ \\
Z score of lumbar spine BMD & $-1.14 \pm 0.14^{\mathrm{c}}$ & $-0.55 \pm 0.15^{\mathrm{b}, \mathrm{e}}$ \\
T score of radius BMD & $-5.51 \pm 0.60$ & $-4.57 \pm 0.54$ \\
T score of lumbar spine BMD & $-2.83 \pm 0.24^{\mathrm{d}}$ & $-2.09 \pm 0.26^{\mathrm{a}, \mathrm{f}}$ \\
\hline
\end{tabular}

All data are presented as the mean \pm SEM. a: $\mathrm{P}<0.05$, compared to preoperative values. b: $\mathrm{P}<0.01$, compared to preoperative values. c: $\mathrm{P}<0.05$, compared to $\mathrm{Z}$ score of preoperative radius $\mathrm{BMD}$. $\mathrm{d}: \mathrm{P}<0.01$, compared to $\mathrm{T}$ score of preoperative radius $\mathrm{BMD}$. e: $\mathrm{P}<0.05$, compared to $\mathrm{Z}$ score of postoperative radius $B M D$. $\mathrm{f}: \mathrm{P}<0.01$, compared to $\mathrm{T}$ score of postoperative radius $\mathrm{BMD}$. 

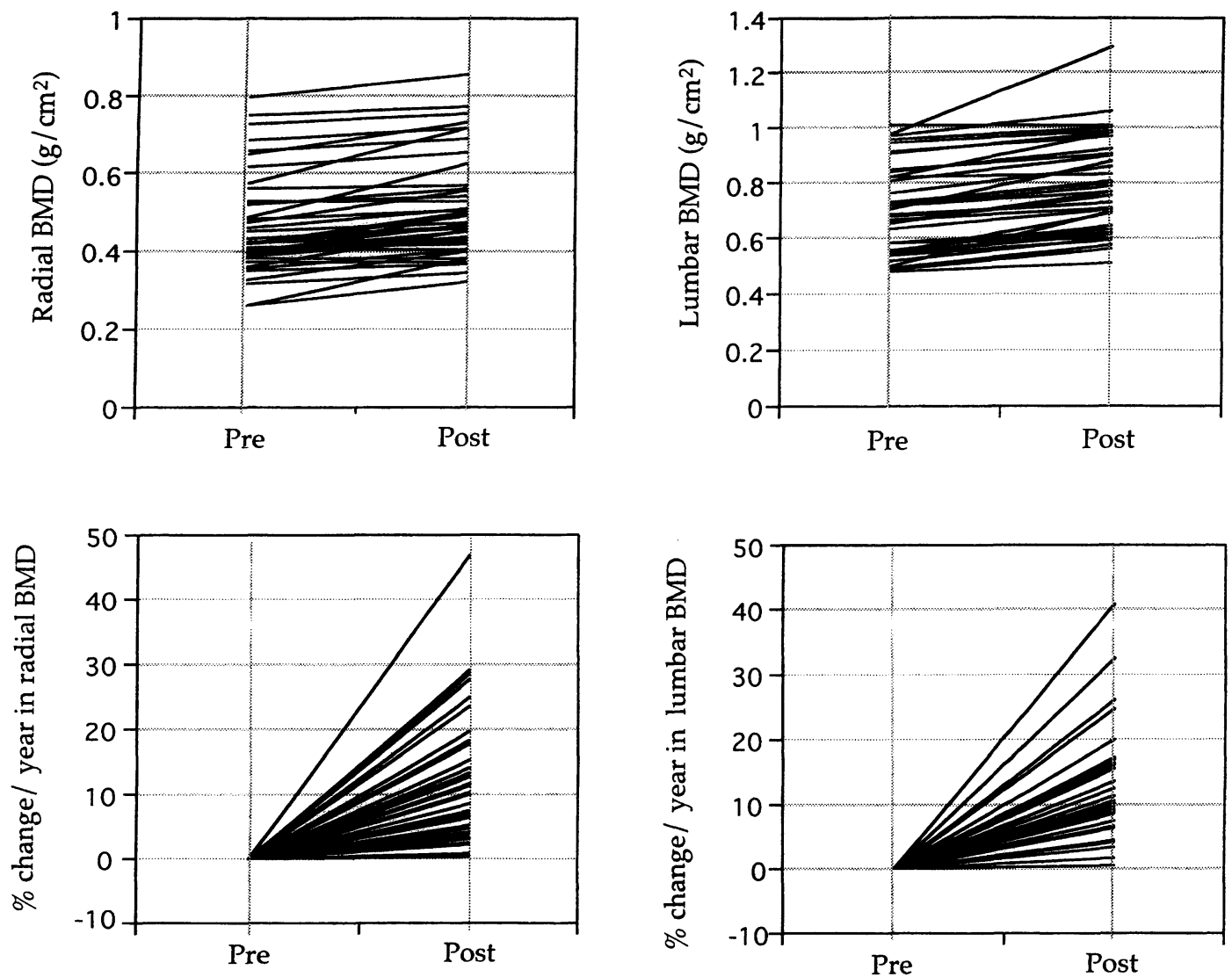

Fig. 1. BMD changes from preoperative to 1 year-postoperative states in individual patients. "Pre", preoperative BMD values; "Post", 1 year-postoperative BMD values.

Table 2. Correlation coefficients between annual percentage and net changes in BMD versus those in intact PTH and ALP after parathyroidectomy

\begin{tabular}{lcccc}
\hline & \multicolumn{2}{c}{ Intact PTH } & \multicolumn{2}{c}{ ALP } \\
\cline { 2 - 5 } & \% change & net change & \% change & net change \\
\hline \% change/year in radial BMD & -0.101 & -0.013 & -0.183 & $-0.294^{\mathrm{a}}$ \\
net change/year in radial BMD & -0.002 & -0.037 & -0.117 & $-0.551^{\mathrm{b}}$ \\
$\%$ change/year in lumbar BMD & -0.335 & -0.079 & $-0.514^{\mathrm{b}}$ & $-0.658^{\mathrm{c}}$ \\
net change/year in lumbar BMD & -0.358 & -0.049 & $-0.471^{\mathrm{a}}$ & $-0.692^{\mathrm{c}}$ \\
\hline
\end{tabular}

a: $\mathrm{P}<0.05$, b: $\mathrm{P}<0.01$, c: $\mathrm{P}<0.001$

radial BMD were significantly and negatively correlated only with the annual net change in ALP but not with the percentage change with relatively low correlation coefficients. In contrast, no significant correlations were observed between annual changes in either lumbar or radial BMD and those in intact PTH.

\section{Discussion}

Several densitometric studies in Caucasian pHPT patients have indicated that the cortical as well as cancellous bone reductions are at least partially reversible after PTX with a range of annual increments of 4.0-9.9\%, although they still remained lower than normal for several years after PTX [11- 
16]. The present study on Japanese patients showed that annual increments in both the cortical and cancellous bone mass after PTX were larger than those in any other study previously documented in Caucasian populations, as judged by the percentage recovery of radial or lumbar BMD values $(11.6 \%$ and $12.2 \%$, respectively) (Figure 1). Moreover, although two studies have reported that the percentage increase in lumbar BMD after PTX was greater than that in radial BMD in Caucasian populations [16, 17], our study showed that the extent of BMD increases at the two sites were not significantly different. These discrepancies might arise from racial difference, or might be because our patients suffered from more severe reduction in radial bone mass than their Caucasian counterparts did, as judged by comparison of background BMD parameters documented in the previous studies. The finding of a more pronounced increase in radial BMD values in a subset of patients with lower preoperative values at this site in several studies [14-16] would support the latter possibility.

pHPT is a relatively common endocrine disease with few overt manifestations, presenting most often as asymptomatic hypercalcemia [1]. Conservative management of patients with mild pHPT is not associated with progression of the disease, as reflected by long-term stable biochemical and bone densitometric indices [20, 21]. A major challenge in the management of this disease is how to make the decision as to which patients should undergo PTX. Mole et al. have reported that differences in BMD between conservative management and surgical treatment were small, and that concern about future osteoporotic fracture should seldom be a major factor for surgical correction of mild pHPT [22]. However, the NIH Consensus Development Conference proposed the guidelines for the indication of surgery for pHPT, which included marked cortical bone loss [23], and Silverberg et al. have recently proposed that reduced cancellous bone density is also an indication for surgery in pHPT, since PTX markedly improves lumbar spine BMD in such patients [24]. In this context, our data showing remarkable postoperative increases in radial and lumbar BMD even in patients without osteopenia would suggest a possible indication for PTX in virtually all pHPT patients regardless of their preoperative BMD values.

The present study revealed that the decrease in radial $B M D$ value was more prominent than that in lumbar BMD in pHPT patients before PTX (Table 1). Since the cortical and cancellous bones are predominant at the distal one third of the radius and at the lumbar spine, respectively, these data confirm the previous observation that the cortical bone mass is preferentially reduced in untreated pHPT patients $[2,3]$. Moreover, serum levels of intact PTH and ALP were negatively correlated with $\mathrm{Z}$ score of the radial $B M D$, but not with that of the lumbar BMD in preoperative patients. These findings suggest that the cortical bone is more susceptible to sustained endogenous PTH excess and high turnover rate than the cancellous bone is in untreated patients.

In contrast, the percentage and net changes in the lumbar BMD were significantly and negatively correlated with those in ALP with high correlation coefficients, but those in radial BMD were significantly and negatively correlated only with the annual net change in ALP but not with the percentage change (Table 2). Since serum ALP is a marker for indicator of bone turnover rate, these findings suggest that the postoperative normalization of a higher preoperative bone turnover rate potentiates an increase in cancellous bone mass rather than in cortical one. Alternatively, it has been hypothesized that changes in circulating PTH levels, typically observed in the intermittent administration of exogenous $\mathrm{PTH}$, are important to the anabolic action of this hormone on the cancellous bone $[6,7,25]$. That is, it is possible that an abrupt reduction in the level of circulating PTH after PTX might exert an anabolic effect on the cancellous bone in our subjects. However, since the postsurgical percentage change or net changes in either radial or lumbar BMD were not correlated with those in intact PTH (Table 2), this would suggest that the latter explanation is less likely.

In conclusion, we showed that PTX in Japanese pHPT patients causes dramatic increases in both the cortical and cancellous bone mass even in a subset of patients without overt osteopenia, and that these two bone structures may react differently to a preoperative endogenous PTH excess and a high bone turnover rate as well as to the postoperative normalization of a bone turnover rate. 


\section{Acknowledgment}

This work was supported in part by the Research
Society for Metabolic Bone Disease and Health Sciences Research Grants.

\section{References}

1. Silverberg SJ, Bilezikian JP (1996) Evaluation and management of primary hyperparathyroidism. J Clin Endocrinol Metab 81: 2036-2040.

2. McDermott MT, Perloff JJ, Kidd GS (1994) Effects of mild asymptomatic primary hyperparathyroidism on bone mass in women with and without estrogen replacement therapy. J Bone Miner Res 9: 509-514.

3. Silverberg SJ, Shane E, de la Cruz L, Dempster DW, Feldman F, Seldin D, Jacobs TP, Siris ES, Cafferty M, Parisien MV, Lindsay R, Clemens TL, Bilezikian JP (1989) Skeletal disease in primary hyperparathyroidism. J Bone Miner Res 4: 283-291.

4. Seeman E, Tsalamandris C, Bass S, Pearce G (1995) Present and future of osteoporosis therapy. Bone 17: 23S-29S.

5. Wronski TJ, Yen CF, Qi H, Dann LM (1993) Parathyroid hormone is more effective than estrogen or bisphosphonates for restoration of lost bone mass in ovariectomized rats. Endocrinology 132: 823-831.

6. Tam CS, Heersche JN, Murray TM, Parsons JA (1982) Parathyroid hormone stimulates the bone apposition rate independently of its resorptive action: differential effects of intermittent and continuous administration. Endocrinology 110: 506-512.

7. Reeve J, Meunier PJ, Parsons JA, Bernat M, Bijvoet OL, Courpron P, Edouard C, Klenerman L, Neer RM, Renier JC, Slovik D, Vismans FJ, Potts JT, Jr. (1980) Anabolic effect of human parathyroid hormone fragment on trabecular bone in involutional osteoporosis: a multicentre trial. $\mathrm{Br}$ Med $\mathrm{J}$ 280: 1340-1344.

8. Hodsman AB, Steer BM, Fraher LJ, Drost DJ (1991) Bone densitometric and histomorphometric responses to sequential human parathyroid hormone (1-38) and salmon calcitonin in osteoporotic patients. Bone Miner 14: 67-83.

9. Christiansen P, Steiniche T, Vesterby A, Mosekilde L, Hessov I, Melsen F (1992) Primary hyperparathyroidism: iliac crest trabecular bone volume, structure, remodeling, and balance evaluated by histomorphometric methods. Bone 13: 41-49.

10. Parisien M, Silverberg SJ, Shane E, de la Cruz L, Lindsay R, Bilezikian JP, Dempster DW (1990) The histomorphometry of bone in primary hyperparathyroidism: preservation of cancellous bone structure. $J$ Clin Endocrinol Metab 70: 930-938.

11. Leppla DC, Snyder W, Pak CY (1982) Sequential changes in bone density before and after parathyroidectomy in primary hyperparathyroidism. Invest Radiol 17: 604-606.

12. Martin P, Bergmann P, Gillet C, Fuss M, Corvilain J, van Geertruyden J (1990) Long-term irreversibility of bone loss after surgery for primary hyperparathyroidism. Arch Intern Med 150: 1495-1497.

13. Martin P, Bergmann P, Gillet C, Fuss M, Kinnaert P, Corvilain J, van Geertruyden J (1986) Partially reversible osteopenia after surgery for primary hyperparathyroidism. Arch Intern Med 146: 689-691.

14. Mautalen C, Reyes HR, Ghiringhelli G, Fromm G (1986) Cortical bone mineral content in primary hyperparathyroidism. Changes after parathyroidectomy. Acta Endocrinol (Copenh) 111: 494-497.

15. Warner J, Clifton-Bligh $\mathrm{P}$, Posen S, McElduff A, Delbridge L, Reeve T (1991) Longitudinal changes in forearm bone mineral content in primary hyperparathyroidism. J Bone Miner Res 6 Suppl 2: S91S95.

16. Silverberg SJ, Gartenberg F, Jacobs TP, Shane E, Siris E, Staron RB, McMahon DJ, Bilezikian JP (1995) Increased bone mineral density after parathyroidectomy in primary hyperparathyroidism. $J$ Clin Endocrinol Metab 80: 729-734.

17. Hesp R, Tellez M, Davidson L, Elton A, Reeve J (1987) Trabecular and cortical bone in the radii of women with parathyroid adenomata: a greater trabecular deficit, with a preliminary assessment of recovery after parathyroidectomy. Bone Miner 2: 301-310.

18. Kobayashi T, Sugimoto T, Chihara K (1997) Clinical and biochemical presentation of primary hyperparathyroidism in Kansai district of Japan. Endocr $J$ 44: 595-601.

19. Frolich M, Walma ST, Paulson C, Papapoulos SE (1990) Immunoradiometric assay for intact human parathyroid hormone: characteristics, clinical application and comparison with a radio- immunoassay. Ann Clin Biochem 27: 69-72.

20. Rao DS, Wilson RJ, Kleerekoper M, Parfitt AM (1988) Lack of biochemical progression or continuation of accelerated bone loss in mild asymptomatic primary hyperparathyroidism: evidence for biphasic disease course. J Clin Endocrinol Metab 67: 1294 1298.

21. Silverberg SJ, Gartenberg F, Jacobs TP, Shane E, 
Siris E, Staron RB, Bilezikian JP (1995) Longitudinal measurements of bone density and biochemical indices in untreated primary hyperparathyroidism. $J$ Clin Endocrinol Metab 80: 723-728.

22. Mole PA, Walkinshaw MH, Gunn A, Paterson CR (1992) Bone mineral content in patients with primary hyperparathyroidism: a comparison of conservative management with surgical treatment. Br J Surg 79: 263-265.

23. National Institutes of Health (1991) Consensus development conference statement. J Bone Miner Res 6
Suppl 2: S9-S13.

24. Silverberg SJ, Locker FG, Bilezikian JP (1996) Vertebral osteopenia: a new indication for surgery in primary hyperparathyroidism. J Clin Endocrinol Metab 81: 4007-4012.

25. Harms HM, Prank K, Brosa U, Schlinke E, Neubauer O, Brabant G, Hesch RD (1992) Classification of dynamical diseases by new mathematical tools: application of multi-dimensional phase space analyses to the pulsatile secretion of parathyroid hormone. Eur J Clin Invest 22: 371-377. 